\title{
Ambient intelligence for quality of life assessment
}

\author{
Antonio Bono-Nuez ${ }^{\mathrm{a}, \mathrm{b},}{ }^{*}$, Rubén Blasco ${ }^{\mathrm{b}}$, Roberto Casas ${ }^{\mathrm{a}, \mathrm{b}}$ and Bonifacio Martín-del-Brío ${ }^{\mathrm{a}, \mathrm{b}}$ \\ ${ }^{a}$ Electronics and Communications Dept., University of Zaragoza, C. Maria de Luna 1, 50018 Zaragoza, Spain \\ ${ }^{\mathrm{b}}$ Aragón Institute of Engineering Research (I3A), C. Mariano Esquilor s/n, 50018 Zaragoza, Spain
}

\begin{abstract}
We are often, consciously or unconsciously, self-assessing our quality of life in order to make decisions about our future actions. People with special needs are sometimes not able to perform this evaluation, this being the responsibility of their relatives or carers. The literature shows this to be a challenging task due to the inherent subjectivity, and the limited data collection tools and biased information available. This paper proposes that context awareness and artificial intelligence can support this task by providing digested and objective information about a person's quality of life evolution. Ambient Assisted Living continuously obtains relevant data from different sources such as sensors, the use of household appliances and interaction with user interfaces. An artificial neural network model known as self-organizing maps processes this data to monitor how the user carries out different activities of daily living (e.g. cooking or doing the washing). This information, together with statistical analysis from the said data, is automatically compiled by the system in a report to visualize trends in user behavior that might lead to the detection of a person's cognitive, physical or sensory deterioration. This report has been validated by a group of experts who considered it a tool of great usefulness and power to complement existing tools used by social workers.
\end{abstract}

Keywords: Ambient intelligence, ambient assisted living, quality of life evaluation, self-organizing maps

\section{Introduction}

Population ageing is a reality in all developed countries. Population projections for future years are alarming; e.g., for the European Union the ratio of people aged 65 years or over will increase from $17.1 \%$ to $30.0 \%$ in 2060 [22]. This situation poses a challenge to society and the social services, which could be overwhelmed by the growth of the elderly population.

In this context, technology can play a relevant role providing cost-effective solutions for improving care for the elderly and people with disabilities in a nonintrusive way, improving their independence and health, and preventing social isolation [10]. It poses an alternative to hospitalization or institutionalization, allowing the elderly and/or disabled to be cared for over a longer period in their own environment at home.

Ambient Intelligence (AmI) is especially powerful, enabling integral solutions for supporting the inde- pendent life of a person. This specific AmI application is known as Ambient Assisted Living (AAL) [1].

Usually in an AAL system, sensors collect context information, an intelligent tool processes the data, actuators provide context action and user interfaces interact with the user. AAL systems commonly need to handle great amounts of low level data about the user, his/her behaviour and the environment, in order to support the decision system.

In this paper we propose to use this information in order to support the work of caregivers by periodically providing them with objective information in the form of a report about the user Quality of Life (QoL). This report is automatically generated by the system processing the data gathered by the smart environment during a period of time; for instance, every month.

Relatives and social workers usually need to observe the person's well-being in order to make decisions about caring actions. Their decisions are actually based on observation, personal interviews, by using accepted and validated methods such as ques-

\footnotetext{
${ }^{*}$ Corresponding author. E-mail: antoniob@unizar.es.
} 
tionnaires, or other more complex metrics such as the International Classification of Functionalities (ICF) [59]. In this context AmI emerges as a powerful tool, pre-processing and objectivizing data to support caregivers' decisions. This information will be objective and prevents the so-called 'Hawthorne effect'; i.e. the user's behaviour changes because he/she knows that it is being studied.

This paper shows the QoL Evaluation System (QoLES) developed in the context of a European funded project [11] whose main objective was increasing elderly and disabled people's autonomy in carrying out their everyday activities, focusing on the work in the kitchen.

The paper is structured as follows. In Section 2 we show a summary of the state of the art of related research. In Section 3 we describe the Smart kitchen architecture, and we contextualize our QoLES. In Section 4 we show the QoLES rationale. The concept and relevant data for the QoLES are validated by a panel of socio-health professionals. In Section 5 we describe the design and implementation of our QoLES, which is based on the Self-organizing map, an artificial neural network used for pattern recognition and data visualization. In Section 6, the QoLES validation is shown and the structure of the reports automatically generated by the tool for the carers is described. Finally, in Section 7 some conclusions of our work are provided.

\section{Related work}

Measuring quality of life is a difficult task because it is a multidimensional and inherently subjective concept that is applied to different people in many different situations.

Tools used to measure quality of life are usually survey based, mainly in the form of questionnaires of two types: generic and disease-specific. Generic ones include WHOQOL questionnaires of the World Health Organization [60], MOS SF-36 Health Survey [49,57], EQ-5D of the EuroQol Group [20], Nottingham Health Profile (NHP) [51], Sickness Impact Profile (SIP) [4,5] and Quality of Well Being SelfAdministered (QWB-SA) Scale [28,29,45], among others.

Disease specific questionnaires are related to widespread specific diseases. Some examples are the EORTC QLQ-C30 that assesses the quality of life of cancer patients [21], Oral Health Impact Profile (OHIP) [35], Visual Functioning Questionnaire
(VFQ-25) [41], Minnesota Living with Heart Failure Questionnaire (MLHFQ) [39] or the Chronic Respiratory Questionnaire (CRQ) [58].

On the other hand, in the research world, there are several studies about measuring and improving quality of life. Some of them are about monitoring and processing information for a specific problem, like coronary artery diseases [19], localization control [53], vital signs monitoring [50], Alzheimer [14], Irritable Bowel Syndrome [43] or dementia [30].

Others are focused on a conceptual approach for measuring and improving quality of life, like modeling the users [9,23], classifying [43], strengthening [24], or creating new quality of life indicators [8], usually by means of artificial intelligence tools, and justifying that ubiquitous computing and virtual reality can improve quality of life [17].

Nowadays, healthcare is changing: personalized healthcare and distributed point-of-care systems that support seamless transitions between hospitals, clinics, the workplace and home, are a must [42]. Personal and environmental monitoring is growing in importance and there are several studies about monitoring the user's environment for extracting relevant data, like activity recognition in a hospital [2], a rule base to control actuators at home [54], monitoring elderly activity at home by means of cameras and environmental sensors data [25,61], activity monitoring for analysis of daily-living behavior [26] or activity recognition that includes user interaction [12].

Finally, two works should be highlighted. The first one, developed at the Center for TeleInFrastruktur [44], consists of a theoretical framework for a QoL evaluation tool focused on dementia care. The other has been presented by the Proactive Health Group of Intel [13], focusing on social interaction and design of individual devices that assist daily routines for improving QoL. While these works are more similar to our proposal, there are marked differences that will be discussed below (Section 7).

In this paper we design and develop an AAL system that aims to continuously gather data from the context, and process it by means of statistical techniques, pattern recognition and artificial neural networks, to evaluate the quality of life of the user. This expert system will be used by carers or professional assistants as a supporting tool for monitoring and diagnosis. A preliminary version of the designed neural networks can be found in [7], while a whole description, design, implementation, analysis and evaluation is included in the following Sections. 


\section{Smart kitchen architecture}

In this section we describe the architecture of the 'smart kitchen' where the system is implemented. The smart system focuses on supporting the user in his/her daily tasks in the kitchen. The intelligence of the system is centralized in an embedded computer, called the e-Servant, where a Service-Oriented Architecture (SOA) over an OSGi framework (Open Services Gateway initiative) [40] supports the different services developed [46]. This computer centralizes the communication with the kitchen appliances, sensors and user interfaces, as well as acting as a gateway to the outside world.

The system has context awareness through three main sources:

- Kitchen appliances provide information about their status through PLC (Power Line Communication). Also, some appliances, such as the washing machine or the fridge, have been improved by adding RFID (Radio Frequency Identification) readers and door sensors. Consequently, food and garments have been labelled with RFID labels. These labels contain similar information to usual labels. For example, food labels codify information about food type (dairy, fruit, meat, etc.), weight, expiry date, etc., and garment labels about colour, garment type, washing and ironing recommendations, etc.

- ZigBee sensors distributed around the house (presence, door contact, light, fire, etc.). Also a RFID standalone reader has been placed in the worktop to provide the user with information about a specific product (food or garment with RFID label).

- User Interfaces; TV remote control, portable devices, touch screen and voice.

Figure 1 shows a simplified system architecture.

First, the user profile is set by the user's carer. This profile defines the cognitive level of the user which determines the help level provided by the system, as well as the interaction level of the interfaces and the user's preferences. A set of deterministic logic rules have been defined in order to support the user. These rules, executed by the Logic Unit, have three main functions:

- to facilitate the use of household appliances and provide useful information and warnings about the use of household appliances

- to detect emergency situations and take corrective action when needed

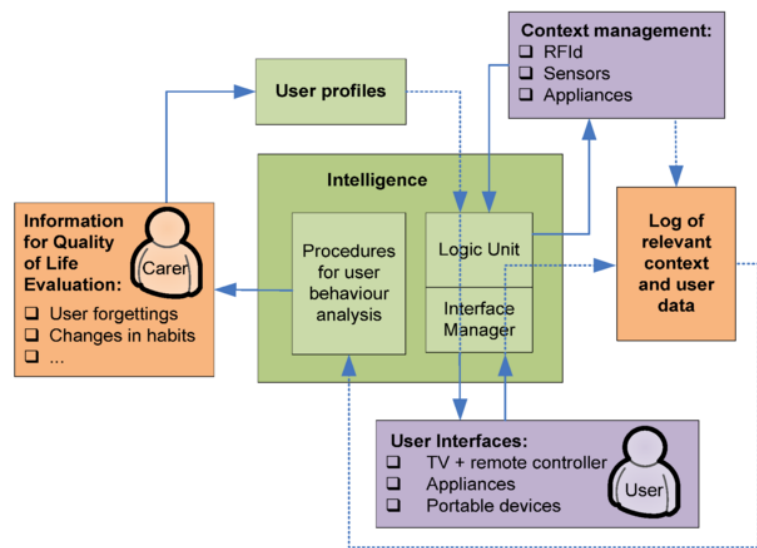

Fig. 1. Smart Kitchen simplified architecture.

- to analyze all the context data to extract relevant information that could be useful for the user's carers and/or relatives in order to evaluate the person's quality of life.

This information is stored in a database and is periodically analysed by the QoL tool which generates a QoL evaluation report for the relatives or user's carers. Our work is focused on the QoL tool that is described in the following Sections.

\section{QoLES rationale}

The first question to answer is if it is possible to study the quality of life (and its evolution over time) from the data retrieved by the AAL system, and what is the most relevant information. It seems evident that changes in the user's habits such as an increase in the number of oversights using the appliances leading to an increase in danger situations, or leaving 'strange' things in the fridge, might be related to changes in the user's cognitive capacity or disorientation. An AAL system is able to gather a huge amount of data about the user that could be used to build a 'snapshot' of his or her present QoL. Thus, based on this idea, our objective has been to create a tool able to process all these data and present useful information for the user's caregiver.

To achieve this objective, a multidisciplinary group of professionals consisting of social workers (3), occupational therapists (8), nurses (3) and engineers (3), most of them experienced with elderly people, have worked closely together to design and validate this concept and to determine which data could be relevant in the kitchen environment. In addi- 
tion, the design of this system is not restricted to the technology and appliances involved in this AAL (not even to existing technology); the QoL tool can be adapted to the technology available.

For the purposes of this study, and in accordance with existing taxonomies of activities of daily living, people's activities in a kitchen are grouped into four areas:

- Food management and storage: including food storage in cupboards, drawers and fridge, outof-date food management, cooling interruption of foodstuffs needing refrigeration.

- Cooking: these activities range from interaction with hobs, ovens and microwaves to actions done in the preparation of the food (following a recipe, cutting, etc.).

- Washing activities: including washing crockery (dishwasher, sink) and clothes (washing machine).

- Other unspecific: this includes all activities performed in the kitchen that are not covered by the previous categories. For example, using the water tap, switching on the lights, interacting with interfaces, throwing the garbage out, etc.

This conceptual phase of the design has been articulated in a two-day workshop. The main focus of the workshop was on elderly people's habits and activities in the kitchen and their relevance in order to detect changes in their daily routines. Brainstorming, polls and debates were the tools used to address the needs, concept and functionalities of a Smart Kitchen.

From the qualitative information gathered in those sessions, a survey about the most relevant parameters to evaluate the cognitive level of an elderly person in the kitchen and his/her loss of capabilities was designed. Responses to the survey were rated between 1 (it is not relevant/not important/not useful) and 5 (it is very relevant/important/useful). 15 surveys were collected and they agreed on the usefulness of a smart system able to evaluate the quality of life of the elderly ( $80 \%$ between 4 and 5), to adapt the appliances to the user's capabilities $(100 \%$ between 4 and 5), to detect changes in the relevant habits (73.3\% between 4 and 5) and to detect loss of abilities $(100 \%$ between 4 and 5$)$. The results of this survey were debated and finally a consensus list of relevant parameters for the QoL evaluation was agreed.

Also, two important conclusions were extracted from the debates in these working sessions. The first is that a smart system would be a useful tool for social workers to complement the information they currently use (from surveys and personal interviews) to assess the user's quality of life. The main benefits detected would be increasing objectivity, data reliability and the amount of data gathered. Personal interviews are influenced by many factors such as empathy between the social worker and the elderly person (which may modify the person's mood and consequently produce a bias) and also dependency on the person's mood variation through the day, week, etc. (observation in an interview is an isolated event in time which may produce a bias).

The second conclusion is that automatic changes in the system's behaviour in order to adapt to the user are not convenient. From the point of view of professionals, this will lead to user disorientation and it is better that the system advises a carer upon the data analysis.

Tables 1-4 outline for each area the high level information that the multidisciplinary group of professionals defined as useful for the Quality of Life Evaluation System (QoLES), as well as the data needed to provide it.

Table 1

Relevant information related with food storage and management

\begin{tabular}{|c|c|}
\hline High-level information & Data needed \\
\hline Changes in shopping habits & $\begin{array}{l}\text { Food tracking in the kitchen (amount, } \\
\text { persistency) } \\
\text { Time between shopping }\end{array}$ \\
\hline Habitual food expiration & $\begin{array}{l}\text { Expiration date (coded in the RFID } \\
\text { labels) }\end{array}$ \\
\hline $\begin{array}{l}\text { Erratic behavior (maybe } \\
\text { due to disorientation or bad } \\
\text { memory) }\end{array}$ & $\begin{array}{l}\text { Number of times the fridge door is } \\
\text { opened without something being } \\
\text { put in or taken out } \\
\text { Erratic cupboard door opening (not } \\
\text { implemented) }\end{array}$ \\
\hline $\begin{array}{l}\text { Failure to attend the } \\
\text { temperature or door alarms }\end{array}$ & $\begin{array}{l}\text { Door and temperature sensor } \\
\text { Number of warnings until warning } \\
\text { is attended }\end{array}$ \\
\hline \multicolumn{2}{|c|}{ Table 2} \\
\hline \multicolumn{2}{|c|}{ Relevant information related with cooking activities } \\
\hline High-level information & Data needed \\
\hline $\begin{array}{l}\text { Absence of mind situations } \\
\text { (changes may indicate losing } \\
\text { of capacities) }\end{array}$ & $\begin{array}{l}\text { Forget the pan on the cooker (e- } \\
\text { Servant could warn after a certain } \\
\text { time) } \\
\text { Wrong stove selection } \\
\text { Liquid overflowing when cooking. } \\
\text { Using an empty pan } \\
\text { Fire or smoke detected by specific } \\
\text { sensors } \\
\text { Forget switching the appliances } \\
\text { off } \\
\text { Number of times the oven and/or } \\
\text { microwave door is open before } \\
\text { ending }\end{array}$ \\
\hline
\end{tabular}


Table 3

Relevant information related with washing activities

\begin{tabular}{ll}
\hline High-level information & \multicolumn{1}{c}{ Data needed } \\
\hline $\begin{array}{l}\text { Erroneous washing machine } \\
\text { operation }\end{array}$ & $\begin{array}{l}\text { Program selected } \\
\text { Identification of the clothes inside } \\
\text { the washing machine }\end{array}$ \\
$\begin{array}{l}\text { Forgetting to take the } \\
\text { crockery/clean clothes out }\end{array}$ & $\begin{array}{l}\text { Number of warnings until crockery/ } \\
\text { clothing is removed }\end{array}$ \\
$\begin{array}{l}\text { Changes in washing } \\
\text { behaviour }\end{array}$ & Time between washings
\end{tabular}

Table 4

Other relevant unspecific information parameters

\begin{tabular}{ll}
\hline High-level information & \multicolumn{1}{c}{ Data needed } \\
\hline $\begin{array}{l}\text { Absence of mind situations } \\
\text { (changes may indicate losing } \\
\text { capacities) }\end{array}$ & $\begin{array}{l}\text { Forget about lights on (presence }+ \\
\text { light sensor) } \\
\text { Erratic movement (position } \\
\text { sensors) } \\
\text { Forget the water-tap on (sound } \\
\text { sensor) }\end{array}$ \\
$\begin{array}{l}\text { Changes in HMI navigation } \\
\text { times }\end{array}$ & $\begin{array}{l}\text { Time needed by the user in each } \\
\text { interaction with the HMI }\end{array}$ \\
\hline
\end{tabular}

\section{QoLES design and implementation}

In this section we show the design and implementation of the quality of life evaluation tool, including the data retrieval process and the pattern recognition algorithms used in our work to process data from sensors. In particular, we present the so-called selforganizing maps (SOM) that are used for QoL monitoring.

\subsection{Context data-retrieval implementation}

Once the high level data useful for QoLES has been defined, we show how our system manages the data. It is possible to identify two levels of data recorded in the e-Servant:

- The Context Event Level, which stores all the events that the e-Servant detects, such as the fridge door being opened or the hob level selected. This kind of event has been defined as low-level because analysis is not required.

- The Logic Unit Event Level, which stores the information processed by high level layers of the system. These data aggregate information relating to appliances, the user and sensor recordings, e.g., the coherence between washing program, clothing colour and temperature selected.
Both Context Events and Logic Unit Events are registered by the e-Servant in a database. These records have the following fields:

- ID: Auto numeric. This field is used as the key.

- Timestamp: day/month/year hour: minutes: seconds.

- Event: kind of event (understandable identification string).

- Provider: Name of the bundle which registers the event.

- Parameters: Text field where the relevant parameters associated to the event are stored.

Table 5 summarizes the high-level events that the e-Servant currently logs.

\subsection{Description of the QoLES}

Regarding the context of the application, the software tool required for this system should have pattern recognition capabilities because the goal is to extract conclusions about user behavior from a large set of sensor data. For instance, from measurable data (e.g. how many times per month the user forgets to switch off a hotplate), the system should detect that the user is undergoing some cognitive deterioration (e.g., oversights increasing).

Regarding the information for consumers (carers and social assistants), the results provided by the system should be easily understood without technical knowledge. Thus, a report providing visual and intuitive results, mainly consisting of graphs, is considered optimum.

For this purpose we make use of Self-Organizing Maps [34], a well-known artificial neural network model used for pattern recognition, exploratory data analysis and for visualizing large data sets. The SOM projects high-dimensional data onto a twodimensional map, providing a visual representation of the problem, which is very useful for decision support systems. From a mathematical point of view, the SOM can be viewed as a nonlinear extension of classical Principal Component Analysis (PCA) and Multidimensional Scaling (MDS) $[6,27,32]$, and it is frequently used for pattern clustering [16].

The SOM has been applied to many problems related to extracting conclusions from large datasets, such as the visualization of massive document collections [31], financial data analysis [48] or video processing [11,52]. In the classical reference [32], there is a comprehensive overview of SOM literature, including hundreds of references to the application of 
Table 5

Summary of the Logic Unit Level Events

\begin{tabular}{|c|c|c|c|}
\hline Event & Description & Parameters & Related technology \\
\hline F_NewItemDetected & $\begin{array}{l}\text { New shop: When a new item or group } \\
\text { of items is added to the fridge, the LU } \\
\text { registers the event } \\
\text { Note that it is only possible when the } \\
\text { food bears a RFID tag }\end{array}$ & New Item list & $\begin{array}{l}\text { Fridge } \\
\text { RFID reader }\end{array}$ \\
\hline F_RemoveTime & $\begin{array}{l}\text { Time between the user being informed } \\
\text { about an expired product and when it is } \\
\text { removed }\end{array}$ & Time (hours) & Fridge \\
\hline F_FoodOutOfDate & Food out of date & Item list & Fridge \\
\hline F_NumberExpiredProduct & $\begin{array}{l}\text { Store the number of expired products in } \\
\text { the last month }\end{array}$ & Month, number of expired products & Fridge \\
\hline F_ErrandBehaviour & $\begin{array}{l}\text { Number of times the fridge door is } \\
\text { opened without something being put in } \\
\text { or taken out in the last month }\end{array}$ & Month, number of times & Fridge \\
\hline F_DoorOpenWarning & $\begin{array}{l}\text { Number of warnings in the last month } \\
\text { and average of reminders }\end{array}$ & $\begin{array}{l}\text { Month, number of warnings, average } \\
\text { of reminders }\end{array}$ & Fridge \\
\hline F_AfterhoursActivity & Fridge afterhours activity detected & - & Fridge \\
\hline WM_Score & $\begin{array}{l}\text { Washing machine score (coherence of } \\
\text { washing program-clothing colour- } \\
\text { temperature) }\end{array}$ & $\begin{array}{l}0=\text { bad selection } \\
1=\text { no appropriate selection } \\
2=\text { suitable selection } \\
3=\text { most suitable selection }\end{array}$ & Washing Machine \\
\hline WM_Washing & Washing & Content & Washing Machine \\
\hline WM_AfterhoursActivity & $\begin{array}{l}\text { Washing machine afterhours activity } \\
\text { detected }\end{array}$ & - & Washing Machine \\
\hline H_LowTimeOn & $\begin{array}{l}\text { Number of times that the hob is switched } \\
\text { on for less than } 10 \text { seconds in a day } \\
\text { Monthly average }\end{array}$ & Month, Time (s) & Hob \\
\hline H_ForgottenOn & $\begin{array}{l}\text { Number of times that the hob is left on } \\
\text { in the last month }\end{array}$ & Month, Number of times & Hob \\
\hline H_TimeHobOn & $\begin{array}{l}\text { Average time that the hob is on in the } \\
\text { morning, afternoon, evening and at } \\
\text { night in the last month }\end{array}$ & $\begin{array}{l}\text { Month, morning avg time on, } \\
\text { afternoon average time, evening } \\
\text { average time, night average time }\end{array}$ & Hob \\
\hline H_AfterhoursActivity & Hob afterhours activity detected & - & Hob \\
\hline K_AfterhoursActivity & Activity in the kitchen afterhours detected & - & PIR \\
\hline HMI_times & $\begin{array}{l}\text { Average navigation times in the } \\
\text { interaction with the HMI for each } \\
\text { scenario in the last month }\end{array}$ & $\begin{array}{l}\text { Month, scenarioID, number of } \\
\text { executions, avg time of navigation }\end{array}$ & $\begin{array}{l}\text { Communication database } \\
\text { analysis }\end{array}$ \\
\hline
\end{tabular}

SOM in very different fields such as speech processing, computer vision, robotics, linguistics, data analysis, etc.

In our work we will use the SOM for monitoring changes in the behaviour and cognitive skills of a person. In the literature there are many references to the use of the SOM for tracking the time evolution of a system, such as the sequential activation of map neurons (nodes). For instance, in [15] a SOM is used for tracking the quality of the service status of communication networks (internet, phone, ...); in [38] a SOM monitors the evolution of a bank, year by year, towards bankruptcy; in [33] the SOM is used for detecting words as phoneme sequences.
A SOM consists of a map of $\mathrm{X} \times \mathrm{Y}$ artificial neurons or nodes, every one computing the Euclidian distance between the input vector presented (sensor data) and the weight vector that every node stores (reference vector). The reference vector stored in every neuron represents a typical pattern. The neuron with the lowest distance is the best matching unit. This neuron will be activated because it has recognized the input pattern (this reference vector is more similar to the set of sensor data presented).

The SOM has no initial knowledge; it must be trained with data (Fig. 2). A comprehensive set of learning patterns (input data) is needed; in our case, a 


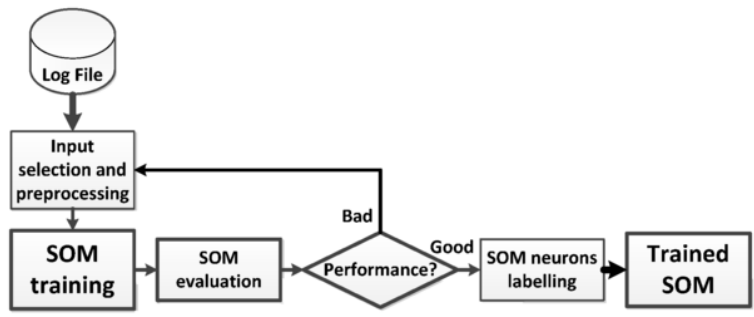

Fig. 2. SOM training phase (development phase).

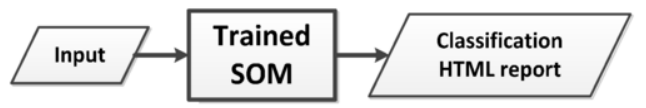

Fig. 3. SOM execution phase.

multidisciplinary team selected input variables, as explained in Section 4.

Once the input data has been gathered, every input variable is usually scaled in the range 0 to 1 (although, depending on the problem, other data preprocessing can be applied).

Next, map dimensions must be established. As indicated above, a SOM consists of $\mathrm{X} \times \mathrm{Y}$ artificial neurons (nodes) [34]; in data analysis and visualization, normally big maps (with a relatively high number of neurons) are used [32], because this allows delimiting cluster frontiers on the map surface.

Once a map is trained with the selected data (in our case, from real people in diverse situations), and depending on the results, input data can be modified (removing or adding variables, extracting anomalous patterns, ...) to re-train the map until the results achieved are satisfactory.

When the training finishes, the reference vectors will be fixed and the map will be ready to be used normally in the execution phase. In this mode (Fig. 3) the SOM can be used as a diagnostic tool providing a response to new inputs (activating the best-matching unit). The mathematical details of the algorithm are available, for instance, in references [32,34].

The SOM algorithm presents some features that we consider very interesting for this application:

- It provides visual results in the form of maps that are easy to understand for non-technical people, such as caregivers.

- Classical related techniques such as PCA and MDS [6,27,48] are linear, while the SOM makes a non-linear projection, uniformly distributing the processed patterns onto the map surface, facilitating the visualization (see Fig. 4 as an illustration).

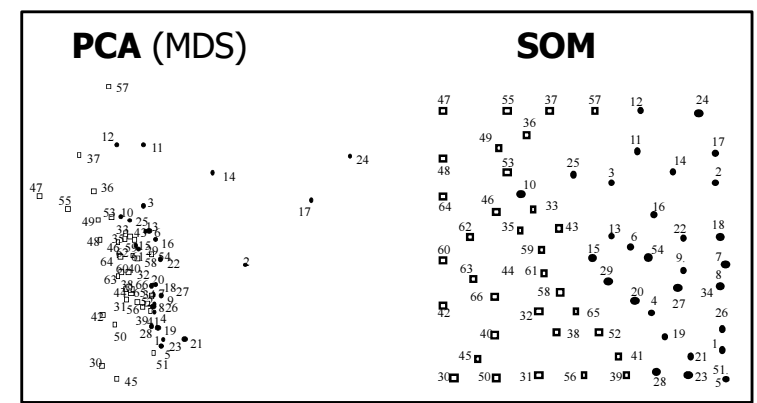

Fig. 4. PCA (first and second principal components) vs SOM for a two-class problem from [48]. PCA makes a linear projection while the SOM makes a non-linear one, uniformly distributing the patterns, facilitating data visualization and delimiting frontiers.

- The SOM is trained with a database, which can be time consuming, but once it is trained, in its normal operation it makes a very simple and fast calculation. Every neuron computes the Euclidean distance between the present input vector and the neuron weight vectors. Then, the neuron with the lowest distance will be depicted on the map (all these operations take less than one second of computer time). In the case of techniques like PCA, when a new input vector is to be processed, it must be incorporated into the database and the elaborate matrix operations required $[6,16]$ must be executed again, which is time consuming for large datasets.

\subsection{SOM design}

Four SOMs have been developed for the study presented in Section 4, each one related to each group of activities (washing, storing food, cooking and unspecific activities). They have been trained with data related to each specific activity in the kitchen. In addition, a 'map of maps' is generated, merging all the activities in only one map ('general map').

As has been described in the Section 5.1, a database contains the data gathered by the AAL system through the Context Event Level (any low level event, i.e. door open/closed, etc.) and the Logic Unit Level (high level data). Some of these data are preprocessed in order to obtain useful input data for the SOM. Table 6 shows the variables finally implemented for each activity area. For example, washing machine variables are obtained using the WM Washing, WM_Score and the door open/closed events.

The final database used for testing the system consisted of 16 input patterns (corresponding to 16 real 
Table 6

Variables used in each map

\begin{tabular}{ll}
\hline Activity area & \multicolumn{1}{c}{ Pre-processed map input } \\
\hline Washing & Time between two consecutive washing cycles \\
& Time from the end of the washing cycle until the user opens the door \\
& Time to take the garments out of the washing machine \\
& Scoring of programming of washing cycles \\
& Time between two consecutive food shopping cycles \\
Food & Time to dispose the garbage \\
Management & Time to detect expired food \\
& Time with the fridge open \\
& Number of times the fridge is opened over the day \\
& Hotplate On and Off in 10 seconds or less \\
Cooking & Hotplate On forgotten \\
& 5 variables related to 5 timeslots: cooker frequency of use \\
& 5 variables related to 5 timeslots: water taps frequency of use \\
& 5 variables related to 5 timeslots: away from home \\
Other unspecific & Lights on without people in room \\
& Time between floor mopping \\
\hline
\end{tabular}

users): 4 with some physical disabilities (mainly osteoporosis and degenerative osteoarthritis), 4 with cognitive disabilities (senile dementia), 4 with visual disabilities (mainly presbyopia) and 4 'normalized' (meaning no disability). A special effort was made to avoid users with multiple disabilities in order to properly validate the tool. This information was recorded during a 2 week period by each real user. In order to expand the data set, we generated more patterns artificially from the 'basic' 16 patterns by adding Gaussian noise (modifying the basic patterns by $15 \%$ of noise) in such a way that more than 100 input patterns were used for training.

The prototype of QoLES has been developed using MATLAB and the SOM Toolbox library [56].

We implemented each of the activities in a separate map; developing 4 maps (see the variables used for each case in Table 6). As an example, we present the implementation process for the food management activity map into the QoLES system.

Once the input data was pre-processed, we trained the map with all available patterns. Figure 5 shows the results. Every component plane (Fig. 5a) represents in shades of grey the distribution of every weight (and corresponding input variable) in every neuron on the map surface. Smooth weight distributions indicate a good training process, which is corroborated by low quantization and topographic errors achieved in the training process by using the SOM Toolbox [56] (common measures of the quality of the generated maps [32]). The combination of the component planes generates the SOM.

Figure $5 \mathrm{~b}$ shows every input (training) pattern labeled onto its corresponding best-matching neuron (the most activated neuron for each input pattern), colored in one of these groups: normalized (green), cognitive disability (red), physical disability (magenta) and sensory disability (blue), following expert recommendations (Table 7). When a pattern can be included in several groups (e.g. physical and sensory disabilities), the SOM positions it on the border of adjacent zones.

After training the SOM, it is necessary to delimit the areas on the map surface, showing different colored clusters relating to the different behavior types (Fig. 5c). In all the maps generated by QoLES we depict four areas with the corresponding colors already mentioned. We can see that the areas are compact; in particular, it is interesting that there is only one 'normalized' zone (green).

In relation to the rest of the maps, the washing activities map and the unspecific activities map present good results similar to those of the food management map. In the case of unspecific activities, it is remarkable how well the SOM dealt with such different data (like frequency of water tap activation, user out of the house, ...) to get a perfectly ordered map.

However, although several maps were developed for cooking activities, we never obtained a correctly trained map. In our opinion, the problem is related to the limited database available for testing the system.

In the last step, a 'general map' is generated by using all the input variables used for the 4 preceding maps (29 variables and 16 base patterns). The map is presented in Fig. 6; the results are good enough, although it is more fragmented than the preceding map because of the heterogeneity of the input data and the limited size of the database. 


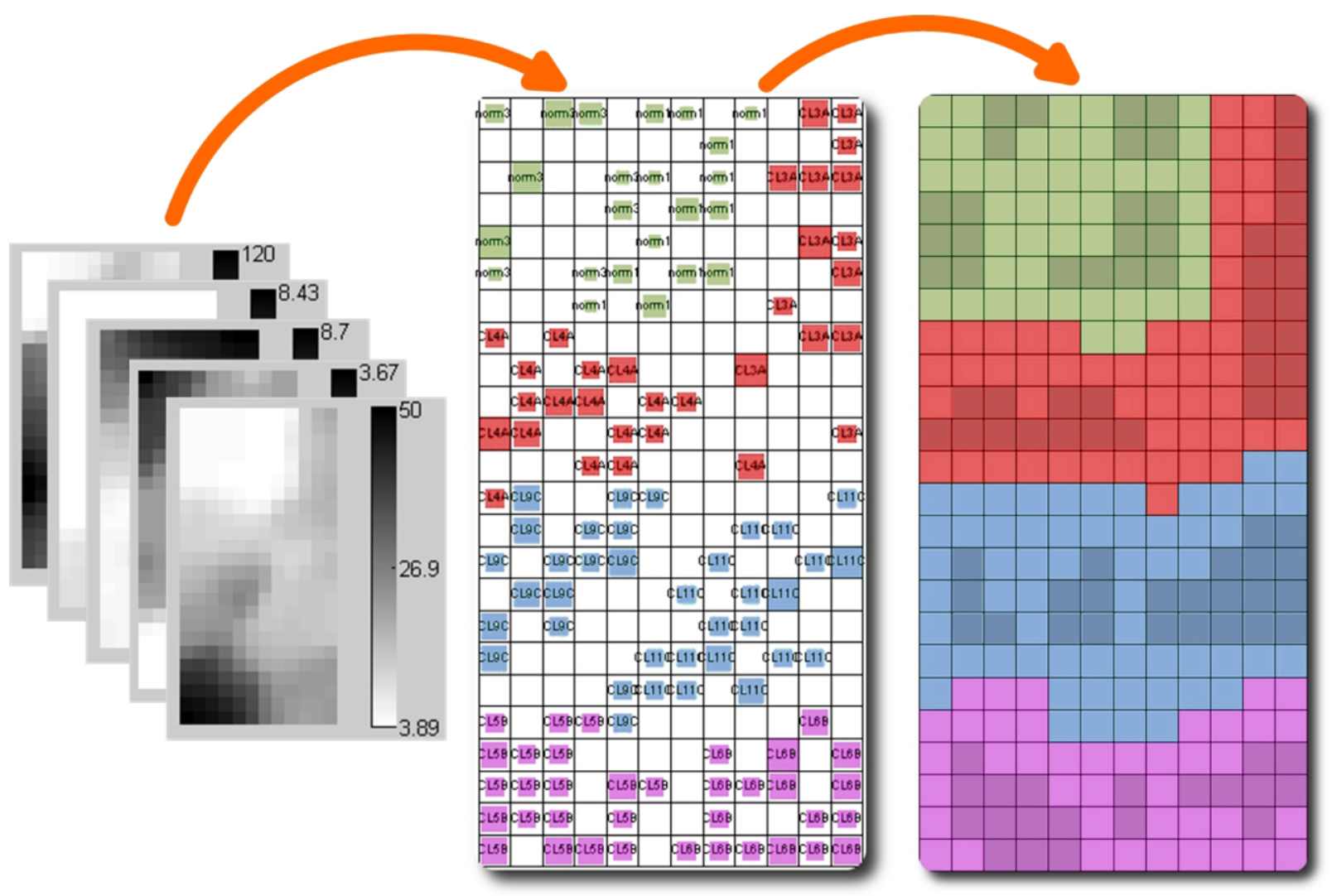

Fig. 5. Food management: a) 5 component planes (weight vector distributions); b) training patterns projections (most activated neuron for every input pattern); c) SOM areas: cognitive (red), physical (magenta) and sensory (blue) disabilities, and normalized (green). (Color figure online)

Table 7

Colours used in the map areas and their relation to different disabilities

\begin{tabular}{lll}
\hline Color & Type of disability & \multicolumn{1}{c}{ Pattern labels } \\
\hline Red & Cognitive & CL1A, CL2A, CL3A, CL4A \\
Magenta & Physical & CL5B, CL6B, CL7B, CL8B \\
Blue & Sensory & CL9C, CL10C, CL11C, CL12C \\
Green & Normalized & Norm1, norm2, norm3, norm4 \\
\hline
\end{tabular}

Thus, in spite of the limited data available for training, the results can be considered valuable because ordered maps are achieved in most cases, with smooth weight distributions and low quantization and topographic errors.

A 'novelty detector' is also implemented [32]. The map in its normal operation computes the Euclidian distance between an input pattern and the weight vector of every neuron. The neuron with the lowest distance (best-matching) is declared 'the winner' (the most activated neuron), being the one that has recognized the input. In normal conditions, this distance must be near zero. Nevertheless, when the smallest of the calculated distances is bigger than a specific thre- shold (fixed in advance), it can be considered that the pattern is anomalous because it does not match well with any training pattern. We experimentally established as anomalous any pattern whose Euclidian distance was 3 times bigger than the mean of the training pattern distances. When an anomalous input vector is repeatedly detected in the on-line (everyday) operation of the system, it is treated as an alarm; the QoLES will send a specific message about this possible anomaly, alerting the carer.

Finally, all this information is incorporated in an easy to read HTML report, especially suitable for non-technical people. 


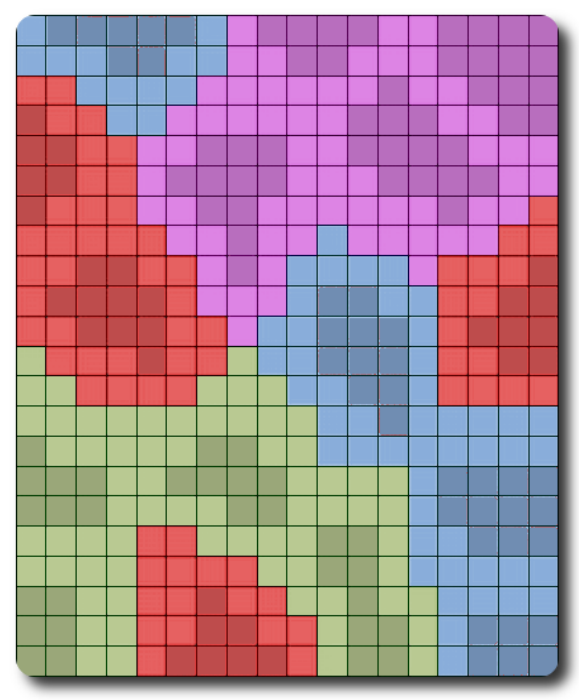

Fig. 6. General map: cognitive (red), physical (magenta) and sensory (blue) disabilities, and normalized (green). (Color figure online)

\subsection{SOM interpretation for QoL assessment}

To be really reliable, all the maps must be trained (Fig. 2) with data from users in very different situations and with different capabilities or skills. When analyzing a map, especially the 'general map' (Fig. 6), it must be considered that a SOM represents a projection of a complex high-dimensional system in only a 2 dimensional map, where every colored square stores a behavior pattern type and nearby squares represent similar patterns. Obviously, this projection process leads to some loss of information or distortion, as occurs when developing a world map, where some regions can be neighbors in reality but can not be so in the map (as occurs with Alaska and Siberia in many world maps). Thus, when SOM areas are evaluated it is common that not all areas have contact with all the others and some cuts or distortions can appear (especially in highly non-linear problems). Nevertheless, the map self-organizes itself to represent the multi-dimensional space as faithfully as possible. In our case (Fig. 6), it is very interesting that the 'normalized' (green) area does have contact with all other areas. It indicates that when a person is well and starts deteriorating, the itinerary can always find a continuous path.

After the training phase, which is done only once (as part of the design of the system), the maps are used in 'normal mode' (Fig. 3): new user data is presented to the tool, every neuron computes the Euclidian distance between its weight vector and the new

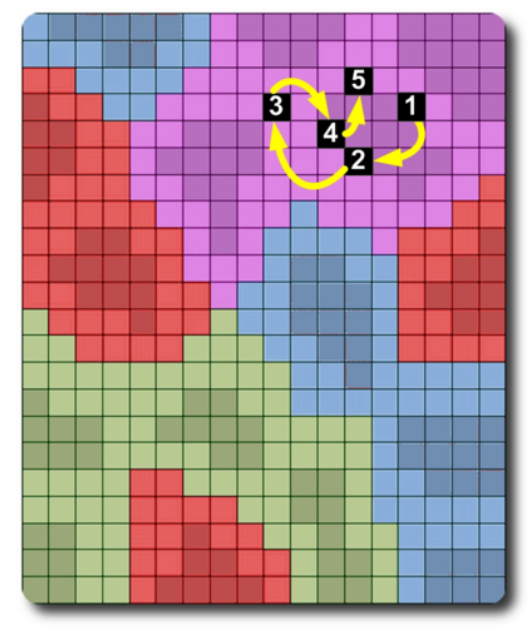

Fig. 7. General map with 'hits' from the same person at different times, appearing in the same area (physical disabilities, magenta): no deterioration. (Color figure online)

input vector, and the neuron with lower distance will be activated, indicating the actual state of the user. The social worker can monitor the evolution of a person by watching the trajectory of the activated squares, or 'hits' after feeding the tool with a series of input patterns recorded from the same user at different times (days, weeks, months or years). The user trajectory could show relevant changes in his behavior, such as cognitive or physical skill loss. This information is useful for the carer in order to see the quality of life evolution and foresee deteriorations.

For example, an old man who limps because of osteoarthritis (physical disability) takes longer to get from one place to another and, therefore, variables such as "Time with the fridge open" and "Time from the end of the washing cycle until the user opens the door" (Table 6) are greater than those of a normalized person. These data are processed by the SOM (Fig. 7) and hits appear in the area of physical disabilities (magenta region). As the elderly man's limp is not getting worse, and he has no symptoms of other disabilities, all the hits are close (no deterioration).

In the second example (Fig. 8), an elderly person without disabilities starts to show symptoms of forgetfulness and disorientation that might indicate senile dementia and even Alzheimer's (cognitive disabilities, red area of the map). This would be detected by increasing values of variables such as "Hotplate on forgotten", "Lights on without people in room", ... (Table 6) which will lead to the trajectory presented in Fig. 8. If a clear shift from the 


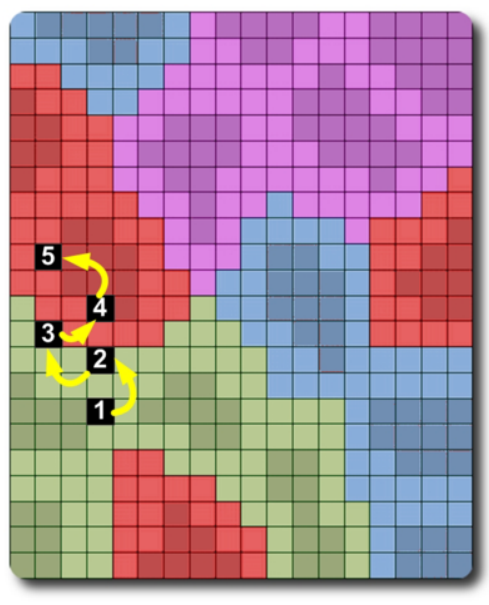

Fig. 8. General map with 'hits' shifting from normalized (green) to cognitive area (red), indicating possible deterioration. (Color figure online)

normalized area (green) to the cognitive disabilities area (red) is presented in consecutive reports, it is possible that the user is experiencing either a real loss of skills or unexpected behavior.

It is possible that an occasional 'hit' appears displaced from its usual area. This is normally due to sporadic behavior (e.g. forgetting to close the fridge) or a system malfunction (e.g. a sensor is not working properly). In this case, the 'novelty detector' will probably indicate that this data is an anomaly and should be treated as such.

These maps, including 'hits' and anomalies, will be used as a support tool for monitoring and diagnosis, and will be included in reports periodically sent to the carers. This process is tackled in the next section.

\section{QoLES validation}

In the execution phase, new patterns recorded from real users are periodically presented to the SOMs (for instance, every week or every month). In every map, the cluster area where the winning neuron is located will indicate to the carers the current situation of the person being monitored. This is the only map-related information that professional assistants need to use.

A report in HTML format is generated periodically and automatically by the QoLES tool (Fig. 9). This report is sent by email to the carer or uploaded to a website which must be accessed with a password.

The report includes:

- A header with user data (name, ID number, etc.).
- Statistics of the last time period (mean deviation, variance, etc.).

- Nutritional information on stored food.

- Laundry statistics.

- A list of possible anomalies (special patterns).

- The different SOMs with the trajectories corresponding to the winning neurons of the last month.

By considering the series of hits on the map surfaces, the carers can evaluate the evolution of the person, forecast deteriorations and make decisions. Figure 9a shows one of the maps with the corresponding trajectories and Fig. $9 \mathrm{~b}$ shows an example of the statistical data provided to the carer.

The final system has been validated by heuristic evaluation, with 31 caregivers participating in two countries (United Kingdom and Spain). 22 of them were professionals (14 nurses, 2 doctors, 2 social workers, 3 occupational therapists and 1 social educator) and the other 9 where relatives of elderly people. The aim was to gather their opinions about the main features of the e-Servant.

This kind of validation, usual in software design [3], is performed by a panel of 'experts' (in this case, professionals and caregivers) when it is not feasible to have a system up in terms of cost and time in order that it may be adjusted (for instance, in our case a large database would be desirable). In the literature there are references to similar problems in software evaluation [3,37], ambient design displays [36] and even devices supporting the elderly [55].

Focusing on the assessment process, caregivers attended a presentation describing the operation of the system and visualized several videos with examples of use. After this, they studied the QoLE reports and discussed the smart kitchen. Finally, they completed a questionnaire with their opinions about the main functionalities of the system. Other generic questions such as the capacities of the system to help the user to carry out his daily activities were also included.

Among ten functionalities of the system evaluated by this procedure, the functionality "detect routine changes in the kitchen to inform whenever there are changes in conduct patterns that can identify any loss of abilities in the user" was the second most valued, obtaining an average score of 4.33 (over 5). Only the "Trigger emergency warnings (fire, smoke, flood) and act in case there is no response" functionality achieved a higher rate (4.76).

Most of the caregivers agreed that the smart kitchen will help the person to carry out daily activities 
WASHING SOM: Washing Machine related Hits:

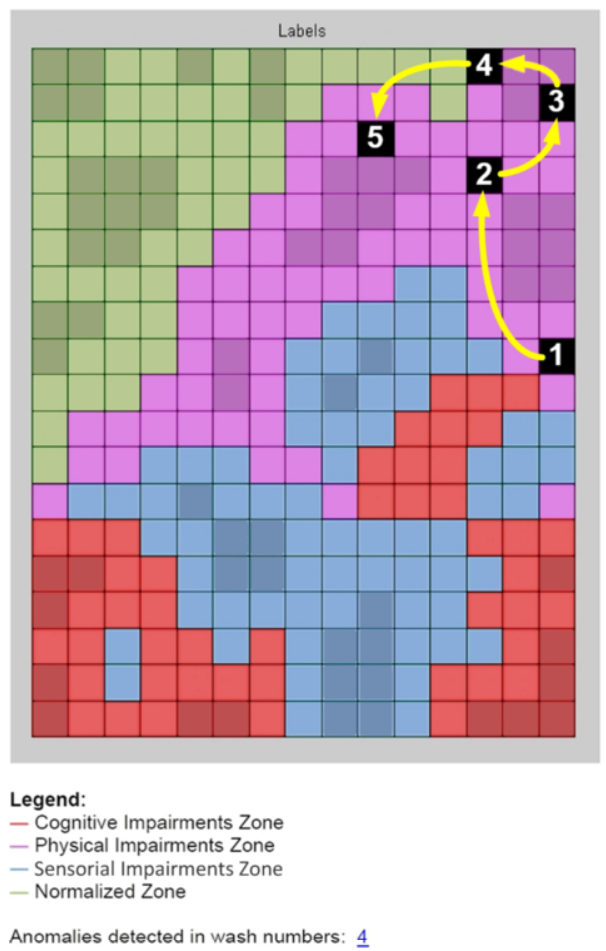

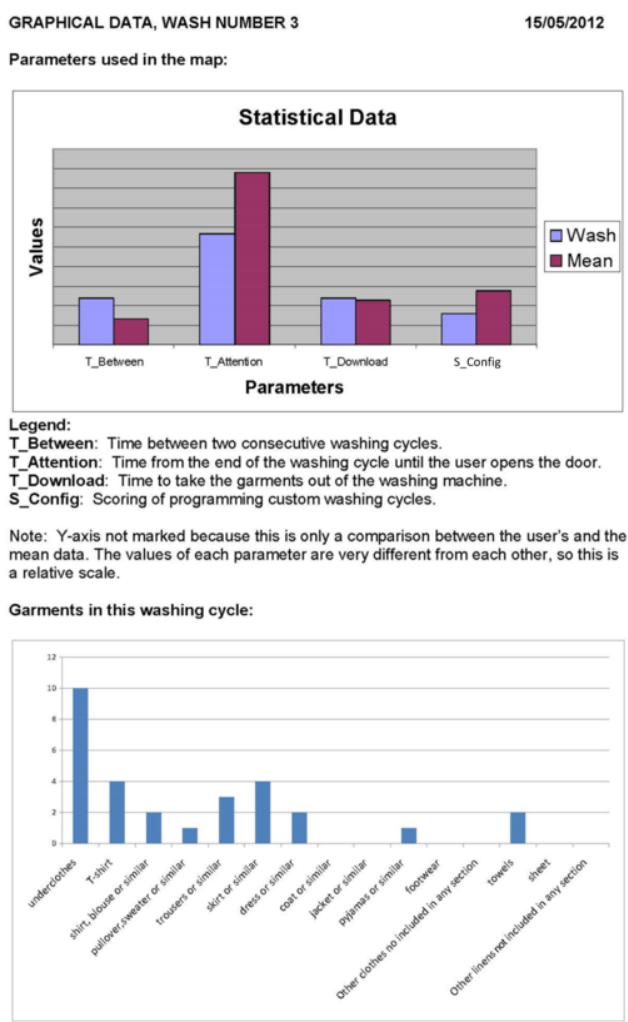

Fig. 9. Sample pages of the report in HTML format sent to carers: a) Washing map with hits b) Statistical information.

(3.38), that it can increase the quality of life of the person (3.35) and that the system can contribute to the autonomous life of the person (3.32). Analyzing the qualitative data recorded in the debates, caregivers considered the report generated by the QoLES (Fig. 9) as really useful, given its capacity to complement traditional tools and to provide information in a concise, simple and visual way.

Additionally, this work, developed within the framework of a European Project [18], was assessed by three experts with many years of experience evaluating research projects relating to Ambient Assisted Living applications. All of them agreed on the remarkable novelty, interest and potential of this tool for the support of elderly people.

\section{Conclusions}

In this paper we describe the Quality of Life Evaluation System developed within the framework of the European funded project Easy Line+ [18]. This tool automatically presents high level information from low level data provided by a smart environment hav- ing several sensors, smart white goods and multimodal user interfaces installed in a real apartment.

This high level information takes the form of a report that is periodically and automatically sent to carers for monitoring the QoL state of elderly people or people with disabilities who live in the apartment. One central element of the report is self-organizing maps that visually provide the current QoL state of the person, showing trends in the form of trajectories over the maps. Interpretation of this information enables the detection of any deterioration in the user's cognitive, physical or sensory conditions.

The main advantage over traditional methodologies for QoL assessment is enhanced objectivity based on continuous data retrieval. In any case, the tool is not a substitute for personal interaction or human observation, but it is a powerful complement.

A heuristic evaluation of the tool has been carried out by a group of 22 professionals and 9 family carers. The experts recognize the interest and capacity of the system as a supporting tool for monitoring and diagnosis.

We think that our approach is novel compared to other approaches reported in the literature. In Sec- 
tion 2 we referenced the works [44] and [13] as the most similar. On the one hand, our approach is different from [44] which is based on a theoretical framework focused on dementia, while our work encompasses all disabilities in general, and our system has been completely developed and built. On the other hand, regarding [13], our system is a kind of general solution (it does not need to be adjusted to particular users), unlike Intel's proposal [13] that focuses on specific solutions for specific users (social interaction for lonely people and personal devices for elderly people with Alzheimer's).

Finally, possible future work would include extending the proposed system to the rest of the house, including new sensors and incorporating mobile devices in the system.

\section{Acknowledgements}

This work was supported by the European Commission under the Easyline+ project (project no.: INFSO-IST-045515). The authors want to thank all the partners involved in the development of the Easyline+ project, and especially Dr. Armando Roy.

\section{References}

[1] E. Aarts and R. Wichert, Ambient intelligence, in: Technology Guide, H. Bullinger, ed., Springer, Berlin, Heidelberg, 2009, pp. 244-249.

[2] M. Aminian and H.R. Naji, A hospital healthcare monitoring system using wireless sensor networks, J. Health. Med. Inform. 4(121) (2013), 2.

[3] S. Beecham, N. Carroll and J. Noll, A decision support system for global team management: Expert evaluation, in: IEEE Seventh International Conference on Global Software Engineering Workshops (ICGSEW), 2012, pp. 12-17.

[4] M. Bergner, R.A. Bobbitt, W.B. Carter and B.S. Gilson, The sickness impact profile: Development and final revision of a health status measure, Medical Care 19(8) (1981), 787805 .

[5] M. Bergner, R.A. Bobbitt, S. Kressel et al., The sickness impact profile: A conceptual formulation and methodology for the development of a health status measure, Int. J. Health Serv. 6 (1978), 393-415.

[6] M. Berthold and D.J. Hand, Intelligent Data Anaysis, Springer, 2003.

[7] A. Bono-Nuez, B. Martín-del-Brío, R. Blasco-Marín, R. Casas-Nebra and A. Roy-Yarza, Quality of life evaluation of elderly and disabled people by using self-organizing maps, in: Distributed Computing, Artificial Intelligence, Bioinformatics, Soft Computing, and Ambient Assisted Living, Springer, Berlin, Heidelberg, 2009, pp. 906913.

[8] D. Botteldooren, A. Verkeyn, B. De Baets and P. Lercher, Fuzzy integrals as a tool for obtaining an indicator for quali- ty of life, in: IEEE International Conference on Fuzzy Systems, 2006, pp. 1065-1071.

[9] R. Casas, R. Blasco, A. Robinet, A. Roy Delgado, A. Roy Yarza, J. McGinn, R. Picking and V. Grout, User modelling in ambient intelligence for elderly and disabled people, in: Proc. of the 11th International Conference on Computers Helping People with Special Needs (ICCHP 2008), 2008.

[10] M. Chan, E. Campo, D. Estève and J.Y. Fourniols, Smart homes - Current features and future perspectives, Maturitas 64(2) (2009), 90-97.

[11] Q. Chen, K. Kotani, L. Feifei and T. Ohmi, VQ-based face recognition algorithm using code pattern classification and self-organizing maps, in: 9th International Conference on Signal Processing, Beijing, China, 2008, pp. 2059-2064.

[12] Y. Chu, Y.C. Song, R. Levinson and H. Kautz, Interactive activity recognition and prompting to assist people with cognitive disabilities, Journal of Ambient Intelligence and Smart Environments 4(5) (2012), 443-459.

[13] S. Ciroux and H. Pigot, Ubiquitous computing to support older adults and informal caregivers, in: From Smart Homes to Smart Care: ICOST 2005, 3rd International Conference on Smart Homes and Health Telematics, Vol. 15, IOS Press, 2005, pp. 11-22.

[14] J.M. Corchado, J. Bajo and A. Abraham, GerAmi: Improving healthcare delivery in geriatric residences, Intelligent Systems, IEEE 23(2) (2008), 19-25.

[15] R. del-Hoyo, B. Martin-del-Brio, N. Medrano and J. Fernández, Computational intelligence tools for next generation quality of service management, Neurocomputing $\mathbf{7 2}$ (16) (2009), 3631-9.

[16] R.O. Duda, P.E. Hart and D.G. Stork, Pattern Classification, Wiley, 2001.

[17] S. Duval, C. Hoareau and H. Hashizume, Improving quality of life from birth to old age with ubiquitous computing and virtual reality, in: International Conference on Convergence and Hybrid Information Technology, 2008, ICHIT '08, 2008, pp. 371-377, 28-30

[18] Easy Line + project website. 2011, 2011-last update, Easy Line + project website. Available: http://www.easylineplus. $\mathrm{com} /$.

[19] S. Eini, Z. Zeybek, M.M. Ozkan and F. Ahmedi, Evaluation of the quality of life outcomes of women and man with coronary artery disease using fuzzy regression analyses, in: IEEE International Conference on Computational Cybernetics, 2008, ICCC 2008, 2008, pp. 265-268, 27-29.

[20] EuroQol Group, EuroQoL - A new facility for the measurement of health-related quality of life, Health Policy $\mathbf{1 6}$ (1990), 199-208.

[21] P. Fayers and A. Bottomley, On behalf of the EORTC quality of life group and of the quality of life unit, Quality of life research within the EORTC - The EORTC QLQ-C30, European Journal of Cancer 38(4) (2002), 125-133.

[22] K. Giannakouris, Ageing Characterises the Demographic Perspectives of the European Societies, Statistics in Focus, Vol. 72, 2008.

[23] G. González, C. Angulo, B. López and J.L.L. de la Rosa, Smart user models for ambient recommender systems, in: Proc. of International Workshop on Ambient Intelligence and Life, 2005, pp. 113-122.

[24] T. Kakiashvili, W.W. Koczkodaj, P. Montgomery, K. Passi and R. Tadeusiewicz, Assessing the properties of the world health organization's quality of life index, in: Computer Science and Information Technology, 2008, IMCSIT 2008, International Multiconference, 2008, pp. 151-154.

[25] B. Kaluža, E. Dovgan, V. Mirchevska, B. Cvetković, M. Luštrek and M. Gams, A multi-agent system for remote 
eldercare, in: Advances in Intelligent and Soft Computing, Vol. 90, 2011, pp. 33-40.

[26] B. Kaluža and M. Gams, Analysis of daily-living dynamics, Journal of Ambient Intelligence and Smart Environments 4(5) (2012), 403-413.

[27] M. Kantardzic, Data Mining: Concepts, Models, Methods \& Algorithms, Wiley- IEEE Press, 2003

[28] R.M. Kaplan, J.W. Bush and C.C. Berry, Health status: Types of validity and the index of well-being, Health Serv. Res. 11 (1976), 478-507.

[29] R.M. Kaplan, W.J. Sieber and T.G. Ganiats, The quality of well-being scale: Comparison of the intervieweradministered version with a self-administered questionnaire, Psychology \& Health 12(6) (1997).

[30] H. Kima, C.P. Hsiao and E. Yi-Luen Do, Home-based computerized cognitive assessment tool for dementia screening, Journal of Ambient Intelligence and Smart Environments, 4(5) (2012), 429-442.

[31] T. Kohonen, S. Kaski, K. Lagus, J. Salojarvi and J. Honkela, Self organization of a massive document collection, IEEE Trans. on Neural Networks 11(3) (2000), 574-585

[32] T. Kohonen, Self-Organizing Maps, Springer Verlag, Berlin, New York, 2001.

[33] T. Kohonen, The neural phonetic typewriter, IEEE Computer Magazine (1988), 11-22.

[34] T. Kohonen, The self-organizing map, Proc. of the IEEE 78(9) (1990), 1464-1480.

[35] D. Locker, D. Matear, M. Stephens and A. Jokovic, Oral health-related quality of life of a population of medically compromised elderly people, Community Dental Health 19(2) (2002), 90-97.

[36] J. Mankoff, A.K. Dey, G. Hsieh, J. Kientz, S. Lederer and M. Ames, Heuristic evaluation of ambient displays, in: Proc. of the SIGCHI Conference on Human Factors in Computing Systems, 2003, pp. 169-176.

[37] G. Margetis, X. Zabulis, P. Koutlemanis, M. Antona and C. Stephanidis, Augmented interaction with physical books in an Ambient Intelligence learning environment, Multimedia Tools and Applications (2012), 1-23.

[38] B. Martín-del-Brío and C. Serrano, Self-organizing neural networks for data analysis: Some financial cases, Neural Computing and Applications 1 (1993), 193-206.

[39] Minnesota Living With Heart Failure ${ }^{\circledR}$ Questionnaire. Available: http://www.license.umn.edu/Products/MinnesotaLiving-With-Heart-Failure-Questionnaire_Z Z94019.aspx.

[40] OSGi Alliance website. 2012-last update. Available: http://www.osgi.org/Main/HomePage, 2012.

[41] R.K. Parrish, S.J. Gedde, I.U. Scott, W.J. Feuer, J.C. Schiffman, C.M. Mangione and A. Montenegro-Piniella, Visual function and quality of life among patients with glaucoma, Archives of Ophthalmology 115(11) (1997), 1447.

[42] M. Pavel, H.B. Jimison, H.D. Wactlar, T.L. Hayes, W. Barkis, J. Skapik and J. Kaye, The role of technology and engineering models in transforming healthcare, IEEE Reviews in Biomedical Engineering 6 (2013), 156-177.

[43] K.I. Penny and G.D. Smith, The use of data-mining to identify indicators of health related quality of life in patients with irritable bowel syndrome, in: Proceedings of the ITI 2009 31 st International Conference on Information Technology Interfaces, ITI '09, 2009, pp. 87-92, 22-25.

[44] C.B. Peterson, N.R. Prasad and R. Prasad, Framework for dementia quality of life assessment with assistive technology intervention, in: IEEE Proceedings of International Association of Science and Technology for Development
(IASTED) Conference in Biomedical Engineering, 2010 pp. 129-134.

[45] Quality of Well-Being Scale - Self Administered (QWBSA). Available: https://hoap.ucsd.edu/qwb-info/.

[46] A. Roy, R. Blasco, A. Marco, D. Cirujano, R. Casas, A. Roy Yarza and R. Picking, Agent-based AmI system case study: The easy Line+ project, in: Advances in Soft Computing: Trends in Practical Applications of Agents and Multiagent Systems (2010), 157-164.

[47] D. Sanchez, M. Tentori and J. Favela, Activity recognition for the smart hospital, Intelligent Systems, IEEE 23(2) (2008), 50-57.

[48] C. Serrano, C. Mar-Molinero and B. Martin-del-Brio, Topology-preserving neural architectures and multidimensional scaling for multivariate data analysis, in: 1st International Workshop on Neural Networks in the Capital Markets, 1993.

[49] J. Ware Jr. and C. Sherbourne, The MOS 36-item short form health servey (SF-36). Conceptual framework and item selection, Med Care 30 (1992), 473-83.

[50] P. Sungmee and S. Jayaraman, Enhancing the quality of life through wearable technology, Engineering in Medicine and Biology Magazine, IEEE 22(3) (2003), 41-48.

[51] M. Tabali, E. Jeschke, T. Dassen, T. Ostermann and C. Heinze, The Nottingham health profile: A feasible questionnaire for nursing home residents? Int. Psychogeriatr. 24(3) (2012), 416-24.

[52] K. Takahashi and S. Sugakawa, Remarks on human posture classification using self-organizing map, in: 2004 IEEE International Conference on Systems, Man and Cybernetics, 2623-8, The Hague, Netherlands, 2004, pp. 10-13.

[53] T. Tsuboi, T. Shiraishi and N. Komuro, Indoor human positioning tracking technique to support high-quality life, in: ICCAS-SICE, 2009, pp. 5745-5749, 18-21.

[54] A.M. Vainio, M. Valtonen and J. Vanhala, Proactive fuzzy control and adaptation methods for smart homes, Intelligent Systems, IEEE 23(2) (2008), 42-49.

[55] J. Vermeulen, J.C. Neyens, M.D. Spreeuwenberg, E. Van Rossum, D. Hewson and L.P. De Witte, The added value of user involvement during the development of a feedback system regarding physical functioning for community-dwelling elderly people, International Journal of Integrated Care 12(1) (2012).

[56] J. Vesanto, J. Himberg, E. Alhoniemi and J. Parhankangas, SOM Toolbox for Matlab 5. Report A57. Helsinki (2000), Available: http://www.cis.hut.fi/projects/somtoolbox/package/ papers/techrep.

[57] J.E. Ware Jr. and B. Gandek, Overview of the SF-36 health survey and the international quality of life assessment (IQOLA) project, Journal of Clinical Epidemiology 51(11) (1998), 903-912.

[58] P.J. Wijkstra, E.M. TenVergert, R. Van Altena et al., Reliability and validity of the chronic respiratory questionnaire, (CRQ), Thorax 49 (1994), 465-467.

[59] WORLD HEALTH ORGANIZATION (WHO), 2003-las update, ICF Application and Training Tools. Available: http://www.who.int/classifications/icf/icfapptraining/en/index. html, 2013.

[60] WORLD HEALTH ORGANIZATION (WHO). WHOQOL. Measuring the Quality of Life. 1997. Available: http://www. who.int/mental_health/media/68.pdf.

[61] N. Zouba, F. Bremond and M. Thonnat, An activity monitoring system for real elderly at home: Validation study, in: 2010 Seventh IEEE International Conference on Advanced Video and Signal Based Surveillance (AVSS), 2010, pp. 278285. 